\title{
A Topological Approach to Shrinking Higher Dimensions of Space to Observable Space-Time: Can the Dimensional Anisotropy of Space Satisfy Mach's Principle?
}

\author{
Eva Deli \\ Phoenix, AZ, 86032 \\ eva.kdeli@gmail.com \\ ORCID: 0000-0003-1126-1970 \\ James F. Peters \\ james.peters3@umanitoba.ca \\ University of Manitoba, Department of Electrical and Computer Engineering and \\ Department of Mathematics, Faculty of Arts and Sciences, Adiyaman University \\ 02040 Adiyaman, Turkey
}

\begin{abstract}
We create a model universe by equipping a topological surface (system) with compact dimensions insulated by an information blocking horizon. The insulated compact WF can produce entanglement independent of distance. Interaction between the system and the WF changes the curvature of the first and the quantum state (frequency) of the second in an interconnected relationship. Thus, the field curvature measures the evolution of the particle WF as time. Positive field curvature creates pressure, whereas negative field curvature generates a vacuum, satisfying the Borsuk-Ulam Theorem and the Page and Wootters mechanism of static time. The accumulation of pressure or vacuum generates poles with contrasting dimensionalities, two-dimensional black hole horizons (time infinite), and fourdimensional cosmic voids (time zero). The orthogonality of the field and the compact WF give rise to global self-regulation that fine-tunes the cosmic parameters and can promote fractal topology. The four-dimensional vacuum in cosmic voids can produce an accelerating expansion without dark energy. When gravity effects are eliminated, we find a new, so far unexplored, order increasing side of entropy. The verifiable and elegant hypothesis satisfies Mach's principle.
\end{abstract}

Keywords: Mach's principle, GR, Borsuk-Ulam Theorem, Topology, Page and Wootters mechanism, dimensional anisotropy 
Introduction

There is a difference in Newton's bucket's water surface when the water is at rest and when the bucket rotates along a curved surface relative to the stars [1]. Without offering a mathematical argument, Mach noted that a relative rotation produces the forces concerning the mass of the Earth and the distant stars and galaxies, i.e., the global distribution of matter. A body's motion relative to the bulk of matter in the universe generates inertial forces.

In Newtonian physics, the intrinsic state of a particle, i.e., its mass has no immediate connection with its extrinsic state in space and time, i.e., its locus and velocity. The universe's distant matter content cannot cause inertia, provided a priori independence of position ( $\mathrm{x}$ ) and momentum (p) of the particle. Mach was unhappy with the idea of absolute space and sought to define inertia as a dynamical quantity determined by the global distribution of matter $[2,3]$.

Mach's profound insight into Newtonian mechanics' shortcoming inspired Einstein to develop general relativity (GR). Gauge symmetries are indifferent to the location; a global symmetry holds as a local symmetry. The frame of reference does not correspond to a directly observable physical object, and it can be moved around without changing the physical situation. To complete the solution, Einstein had to postulate "boundary conditions" at infinity.

Maxwell's demon is an imaginary creature that can lower entropy by allowing faster particles into another compartment. After a while, the compartment would have slower, cold particles, whereas the other would grow hot. Although Landauer's principle offers a brilliant solution, recent work found a mysterious relationship between order and entropy $[4,5]$. We try to shed new light on this puzzle.

The Page and Wootters mechanism of static time (PaW) arises from the Wheeler-De Witt equation. The physical states of the theory do not evolve in time (i.e., the Hamiltonian of the theory does not generate time translations of the physical states for an external time) [6]. A subsystem in a state corresponding to the time $t$ serves as a clock. We create a clock system satisfying both Mach's principle and PaW [7] by attaching a compact WF to a spatial field. Quantum mechanics and string theory suggest that an information blocking horizon insulates the compact dimensions. Therefore, PaW dictates the WF's independence from time. Time progression is the function of interaction; the spatial field will operate as a clock, tracking the discrete frequency evolution of the WF from time zero in the white holes to time infinity in the black holes.

In the present work, we will examine whether a model system consisting of a spatial field connected to compact dimensions can satisfy Mach's principle and replicates the qualities of our physical world. The compact dimensions represent representing string theory and 
quantum mechanics. We will investigate the evolution of spatial topology via interaction. The Borsuk-Ulam theorem can explain how entanglement generates spatial anisotropy, with implications for entropy, inertia, and Mach's principle. We close with a discussion and summary.

Considerations of String theory

The Page and Wootters mechanism $(\mathrm{PaW})$ establishes a static universe without time evolution (i.e., the Hamiltonian of the theory does not generate time translations of the physical states for an external time) $[8,6,9]$. Energy conservation intuitively supports PaW $[8,10]$, yet the idea remained obscure until a recent experimental proof revived interest in the work $[11,12,9]$. We followed the antipodal dynamics of $\mathrm{PaW}$ with zero time evolution $(\Delta \mathrm{E}=0)$.

$\mathrm{H}$ tot $\mid \Psi i=0$

where $\mathrm{H}$ tot is the global Hamiltonian of the universe and $\mid \Psi \mathrm{i}$ is its state

$|\psi\rangle(\mathrm{t})=\mathrm{U}(\mathrm{t})|\psi 0\rangle$

Although a spatial field (SF) without an internal structure is unstable, introducing an ancillary system creates irreversibility, stabilizing the curvature, as shown by other authors $[13,14]$. We equip a spatial field (SF) with ancillary WF (WF) to create a model universe. Insulating strings by information blocking horizon limits particles into discrete frequencies, and it engenders time independence, as dictated by $\mathrm{PaW}$. The WF remains non-local and reversible.

The compact dimensions (i.e., extra dimensions beyond the observable dimensions are wrapped up or curled up onto themselves as in Calabi-Yau spaces) can form entanglement independent of its spatial coordinates, satisfying quantum mechanics. Two systems separated by an information blocking horizon become orthogonal to each other. The orthogonality between gravity and quantum mechanics causes infinities in calculations. The time-independent compact WF permits entanglement independent of spatial coordinates (Figure 1. top). The ERP pairs can observe conservation principles independent of distance [15].

Entanglement is a local instability, satisfying PaW (Figure 2), which triggers interaction. An interaction term in the Hamiltonian couples a clock spatial field to WF [16, 17], tracking its evolution. In other words, the clock system periodically must interact with the system whose evolution it is tracking. 
Interaction satisfies conservation principles; there is symmetry between the Lagrangian, field curvature (Figure 1. bottom), and the quantum state (frequency). The energy difference between the frequencies must be equal to the Lagrangian of the interaction. Their identity might explain the unexpected connection between the monster group $\mathrm{M}$ and modular functions, specifically the $\mathrm{j}$ function.

Interaction with space involves decoherence, which changes the WF's frequencies (Figure 1) and stabilizes the curvature due to its energy need. Instead of a onedimensional manifold moving in time (worldsheet), the evolution of spatial curvature represents the tick-tack of the particle's WF, i.e., time. Interaction generates local pressure differences while leaving the global state unchanged. The above setup supports quantum mechanics' axiomatic formalization: entangled sister particles satisfy conservation laws during quantum phenomena, such as entanglement or the double-slit experiment (Table I).

\section{Topological considerations}

The compactification of space is bordant to a point [18]. Zeroing (shrinking) entanglement via interaction is an orthogonal transformation that modifies local volume via curvature, forming the cosmos' global topology (Figure 2). Greater quantum frequencies are concomitant with positive curvature (loss of volume), whereas slower frequencies parallel negative curvature (increased volume). Because the compact dimensional frequency defines a specific temperature, it also relates pressure and volume to temperature.

The evolution of the spatial curvature, which measures the evolution of the WF, satisfies the Schrödinger equation [19]. Therefore, the particle vibrations of string theory are related to the field strength (curvature or metric) and represent time. This way, the insulated WF is a necessary requirement in forming gravity as a topological space. The curvature of space, i.e., gravity, can measure the evolution of discrete WF.

Topological spaces are central unifying notions in mathematics and physics, such as gravity. The cosmic microwave background (CMB) represents slight density variations and smooth distribution of a nearly spatially flat early universe [20, 21, 22]. In contrast, empty space dominates throughout the current web-like cosmic structure (Figure 3). Galaxies move towards the densest areas and away from regions with little mass - the voids (relative to the universe's overall expansion) [23].

Most of the universe is filled with cosmic voids, with tens million to hundreds of millions of light-years across $[24,25]$. Voids display less than one-tenth or even smaller matter density abundance than the typical or average cosmic density [26, 23]. The larger supervoids are located in the lowest density regions and remain well-insulated from gravitational structures (Figure 4, orange regions). The density surfaces range from vacuum $-2.3,-1.1,-0.7,0.2$ and 
progress through increasingly dense $1.00,1.25,1.50,1.75,2.00$, and 2.25 [23]. The resulting hierarchical organization represents smooth density transitions (Figure 6). In future work, we intend to provide detailed calculations for the field curvature changes during density transitions.

Vacuums spur accelerating expansion with massive objects following diverging velocity flows, like watersheds [22], by pushing against the highest temperature regions. The interior void flow fields push and drive the galaxies, galaxy clusters, and their lattice-like web apart. A nearly spherically symmetric galaxy outflow forms walls, knots, and immense size filaments [20]. Recent void analysis shows hierarchical structures in different levels, indicating that the nonlinear mass distribution mechanisms for the void size distribution. The void size shows a close parallel with galaxy mass distributions [27].

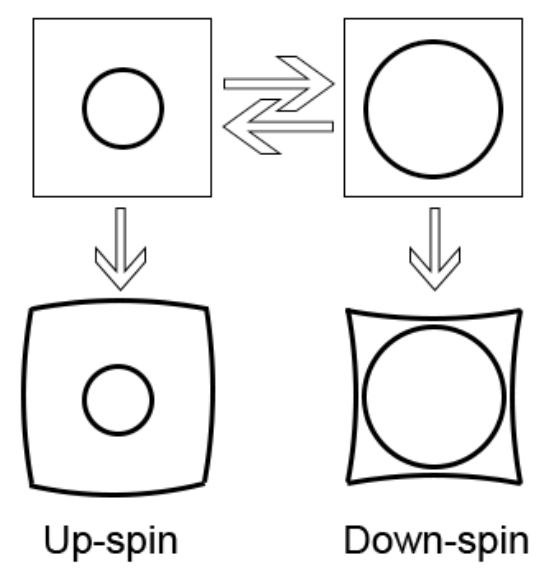

Figure 1. Ancillary clock system in two dimensions Top: Compact dimensions (indicated by circles) are insulated from space by an information blocking horizon, allowing nonlocality, such as entanglement. Bottom: Interaction between the spatial field and the compact dimensions permanently separates the energy function into daughter particles, changing the pressure, which modifies field curvature. 


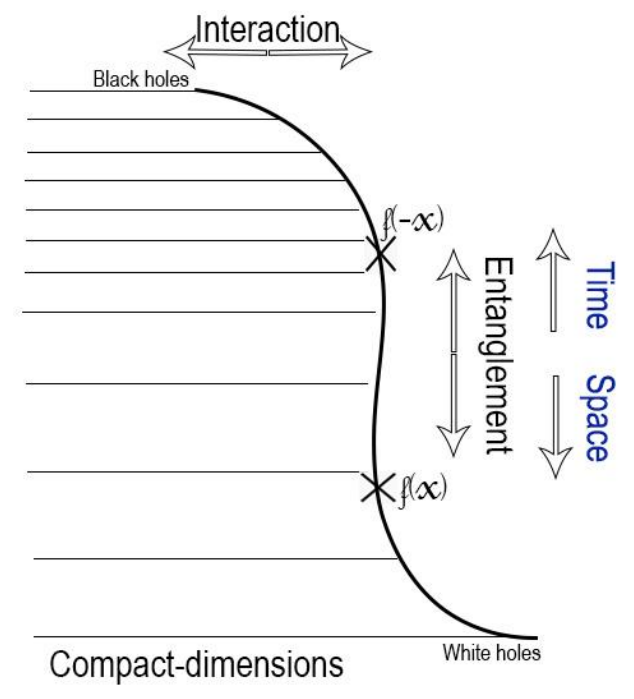

Figure 2. The proposed view of 'space-time' The compact dimensions (horizontal lines) are isolated by an information blocking horizon, limiting particle frequencies to discrete energy levels. Entanglement of WF, $\mathrm{f}(\mathrm{x})$, and $\mathrm{f}(-\mathrm{x})$ evolves the WF and triggers interaction with the spatial field. The field curvature (right curved line) is a clock system that tracks the evolution of discrete compact frequencies by a continuous topology between black holes (top) and cosmic voids (bottom). The field strength increases toward the top.

Entanglement between $f(x)$ and $f(-x)$ engenders differences in field curvature and pressure. Stretching and squeezing points in an antipode-preserving way, their complement can be mapped to the equator. In black holes, time $=\infty$, space $=0$; in cosmic voids, space $=\infty$, time $=0$

Cosmological entropy is related to geometry; it increases with temperature (toward black holes) or with volume (toward white holes) with the poles representing the highest entropy. The expansionary potential of space acts as dark energy, whereas the loss of dimensionality gives the semblance of dark matter. 


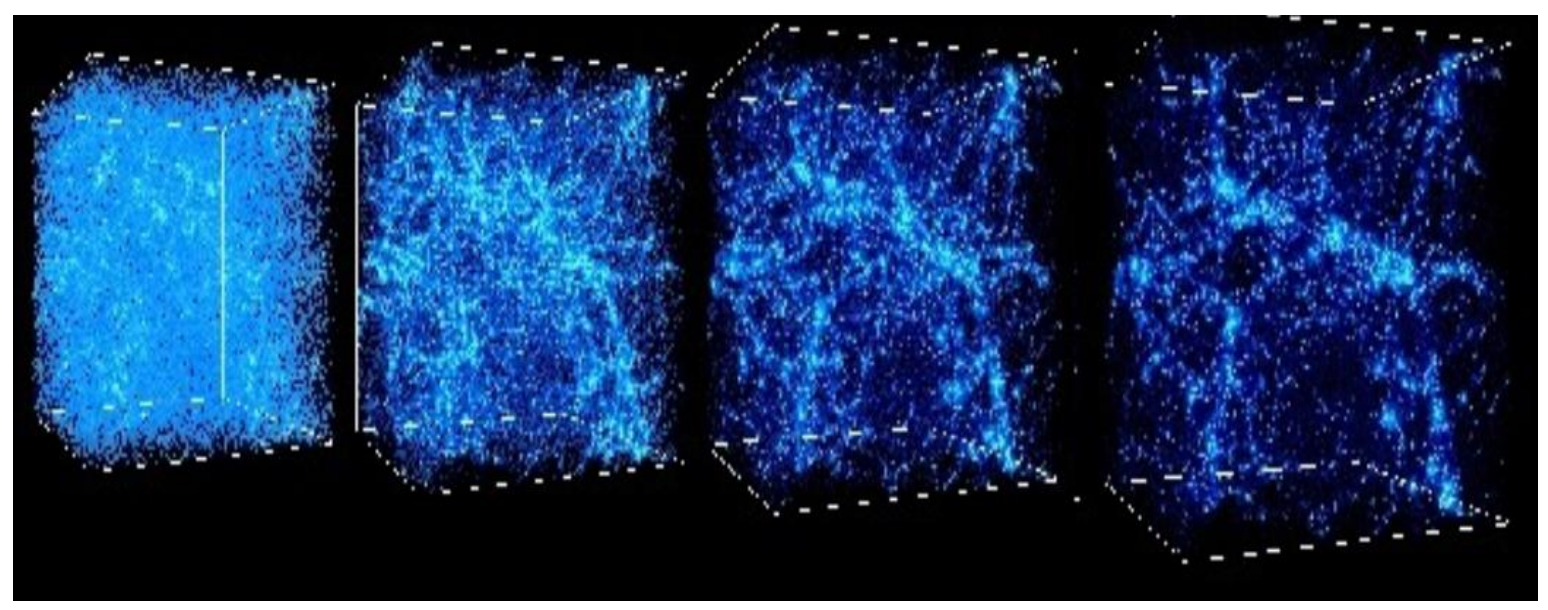

Figure 3. Computer simulation of the cosmic web Thin filaments connect dense, galaxy cluster-filled regions. Voids are nearly empty regions of space. Evolution from left to right shows the gradually increasing curvature differences. Picture credit: Andrey Kravtsov (The University of Chicago) and Anatoly Klypin (New Mexico State University) at the National Center for Supercomputer Applications.

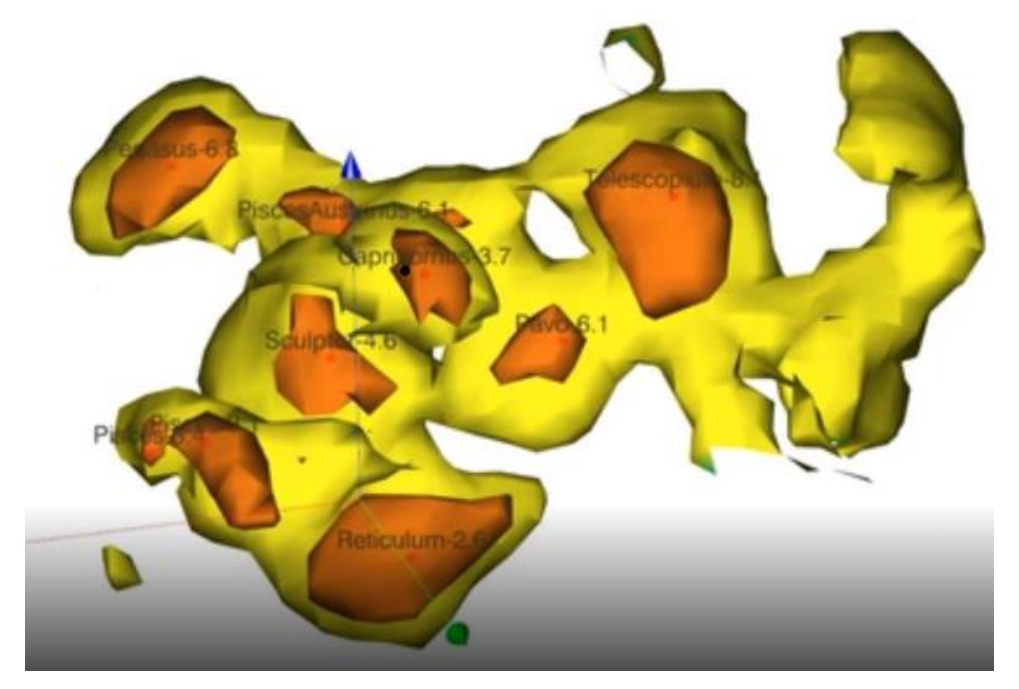

Figure 4. The density map of the Local Void The central void density is -1.1 (orange) and -0.7 (yellow) elsewhere (the negative sign indicates under density). The central location of coldest regions within voids stabilizes them-image from Tully et al., 2019. 
The Borsuk-Ulam theorem (BUT)

In 1961 Rolf Landauer predicted the minimum amount of heat needed to erase a classical bit $E=k_{B} T \ln 2$ (Landauer, 1961), where $\mathrm{k}_{\mathrm{B}}$ is the Boltzmann constant and $\mathrm{T}$ is the temperature of a "reservoir" with which the bit exchanges heat. Because E/T is constant (on a constant temperature), interaction modifies the local volume, which evolves the large-scale structure. Entanglement updates the particles' energy state [9]. The antipodal volume transformations of sister particles update the field curvature [28].

The Borsuk-Ulam Theorem (BUT) states that if an $n$-sphere $S^{n}$ (generalization of a circle) is mapped continuously into an n-dimensional Euclidean space $R^{n}$, there is at least one pair of antipodal points on $S^{n}$ which map onto the same point of $R^{n}$ [29, 9]. Correspondence can be found between a pair of antipodal points, and they can be represented in terms of one point. The analog of this mapping process is the drawing together separate antipodal free particles whose entangled states are correlated in a quantum mechanics view of BUT. This correlation of entangled states can be explained mathematically in terms of the descriptive proximity of a pair of antipodal particles [30, 31]. In other words, thanks to a pair of companionable feature vectors that describe antipodal particles, the particles have a nonempty descriptive intersection $[13,14$ [32]. That is, let $p A, p B$ be a pair of antipodal particles and let be $\Phi(p A), \Phi(p B)$ descriptions of the particles, defined by

$\Phi(p A)=\{x \in p A: \Phi(x)$, a description of $\mathrm{x}$ in $\mathrm{pA}\}$
$\Phi(p B)=\left\{x^{\prime} \in p B: \Phi\left(x^{\prime}\right)\right.$, a description of $\mathrm{x}^{\prime}$ in $\left.\mathrm{pB}\right\}$

For example, $\Phi(p A)$ is a set of descriptions of the parts of $p A$, represented by a feature vector that describes $p A$ with its $n$ parts, namely, $\overrightarrow{\left(\Phi\left(x_{1}\right), \Phi\left(x_{2}\right), \ldots, \Phi\left(x_{n}\right)\right)}$. In the case where a pair of antipodal particles have companionable descriptions, the descriptive intersection $\quad p A \underset{\Phi}{\cap} p B \quad$ is nonempty, i.e., $p A \underset{\Phi}{\cap} p B=\{y \in p A \cup p B: \Phi(y) \in \Phi(p A)$ and $\Phi(y) \in \Phi(p B)\} \neq \varnothing$.

That is, there is at least one component $\mathrm{y}$ in the union $p A, p B$ that has a description $\Phi(y)$ that is common to the descriptions of $\Phi(p A)$ and $\Phi(p B)$, i.e., $\Phi(y) \in \Phi(p A)$ and $\Phi(y) \in \Phi(p B)$. In effect, particles whose states have companionable descriptions can be mapped to a higher state in terms of their descriptive intersection.

From a compactified dimensions perspective, a higher dimension feature vector that describes a physical structure can be shrunk to a lower-dimensional structure by convolving 
(rolling together) the features in a description to a more concise feature vector (Peters J. a., String-Based Borsuk-Ulam Theorem, 2016). According to BUT, the pressure of positive curvature correlates with a negative curvature vacuum. This convolution can be represented topologically by a collection of contraction maps from surface points (each with its own feature vector) to a fixed point, such as a surface centroid with a single feature vector with a reduced number of features. For example, the points on an edge attached between the boundaries of a ribbon $\mathrm{rb} E$ can be mapped to a fixed point $p$ in the intersection of $p$ with the ribbon boundary bdy of the closure cl of ribbon cycle cyc $B$ as shown in Figure 5, using mapping f defined by $f(p \in p q)=p q \cap \operatorname{bdy}(\operatorname{cl}(\operatorname{cycB}))=p$ [33].

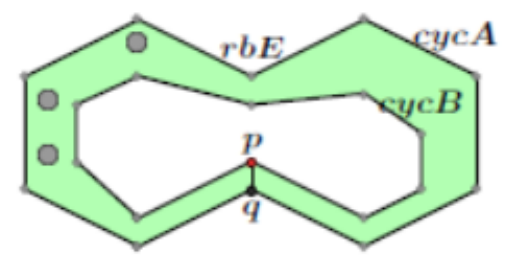

Figure 5. Mapping an edge $p q$ to a fixed point $\mathrm{p}$ on a ribbon boundary

See [34] for further details and mathematical treatment. The notation $n$ within $S_{n}$ stands for an n-sphere [35], an n-dimensional, circular structure embedded in an $n+1$ space [36]. For example, a 2-sphere $\left(S_{2}\right)$ is the 2-dimensional surface of a 3-dimensional space. Antipodal points are, e.g., the poles of a sphere [32]. The mapping $f: S^{n} \rightarrow S^{n}$ is smooth, provided $f$ is a 1-1, continuous, differentiable mapping from the manifold $S^{n}$ (domain of a Euclidean space) into itself in which the inverse mapping $f^{-1}: S^{n} \rightarrow S^{n}$ is also continuously diffentiable [37].

$\underline{\operatorname{deg} f=X^{X} \in f^{-1} \operatorname{sig}(\operatorname{det} \mathrm{dx} f) .}$

The cosmological standard model rests on the assumption that the universe is isotropic around all observers. However, applying conservation principles with BUT show that antipodal changes, separated in space or time, map into one. This spatial and temporal separation results in dimensional anisotropy. Antipodal changes might originate in entanglement and the quantization of particles in quantum mechanics [38].

Recent work on a system consisting of particles of only one type proved the evolution into a system with two types of particles having equal fractions [39, 40]. Moreover, they found the evolution of antipodal transformations, i.e., attraction and repulsion forces.

Because antipodal transformations are matching descriptions mapping into Euclidean space, the lack of volume within positive curvature must compensate for excess volume within 
negative curvature regions (Figure 2 and Figure 6). According to BUT, any two-dimensional black hole boundary (see, e.g., Figure 2) must have at least one antipodal, i.e., a fourdimensional point corresponding to it. Rather than treating the causes of the two regimes independently (dark energy and dark matter), PaW offers a unique cause arising from the same fundamental physical process [41].

\section{Dimensional anisotropy}

Einstein observed the indistinguishability of acceleration and gravity for a person riding in an elevator, despite their irreversible and antipodal thermodynamics. Entropy can prove to be obscure concept occasionally. Time is often represented by a temperature dependent entropy generation and entropy rate $[42,43]$. The evolution of time intervals $(t=1 / v)$ shows a frequency (v) dependence $[44,45]$.

$\mathrm{Ds}^{2}=\mathrm{dr}^{2}+(\mathrm{cdt})^{2}$

The identity:

$1=\cos \psi^{2}+\sin \psi^{2}$

$\sin \psi=\mathrm{cdt} / \mathrm{ds}$

Time emerges from an orthogonal transformation between the compact dimensions and the spatial field, where gravity pushes time forward, acceleration pulls it back (Figure 2; Figure 6). The identical slowing of the clocks caused by the antipodal thermodynamics (gravity and acceleration) suggests their trigonometric origin. Therefore, the Lorentz transformation expresses the sensitivity of the field curvature to quantum frequency changes.

The derivative of the inverse sine function is

$\frac{d}{d \psi} \sin ^{-1} \psi=\frac{1}{\left(1-\psi^{2}\right)}$

Lorentz transformation can be interpreted as the rapidity of curvature change, i.e., the energy input needed for a unit change of curvature (Figure 6).

Landauer's principle intuitively supports the known correlation between information accumulation and high temperatures in black holes [46]. Although black hole physics is a subject of a lot of debate [47], both the AdS/CFT conjecture [48] and the firewall hypothesis [49] support the connections to lesser dimensionality horizons. According to PaW and BUT, any two-dimensional boundary (see, e.g., Fig. 3) has at least one antipodal point pair, i.e., four-dimensional space. 
Antipodal dynamics, such as simultaneous heating and cooling, produce thermal convection cells, and the surface tension in expanding hot gas forms foam [50]. The evolution of oneparticle systems involves naturally arising attraction and repulsion forces and two types of particles having equal fractions $[39,40]$. The reverse energy flow from a colder to a warmer system, such as information backflow, does not violate the Second Law [51, 52]. The twodimensional black hole and four-dimensional white holes represent phase transformation caused by the accumulation of pressure or vacuum, respectively. Gravitational contraction and expansionary vacuum are antipodal.

The cosmological constant problem is the discrepancy between the theoretical vacuum energy density and its empirically measured value [53]. However, vacuum energy might be the function of field strength. If gravitational field effects reduce the measured value of vacuum energy, then spatial expansion is limited to the low field strength of cosmic voids $[54,55]$. Systems with a bounded energy spectrum display negative absolute temperature, and negative pressure akin to dark energy $[56,57]$, cosmic expansion may originate in the zero-point energy of the cosmic voids [58, 55, 19, 59].

The existence of negative temperatures [60, 61, 62, 63, 64, 65] suggests a possible relationship between vacuum energy and temperature [66]. Recent studies show that in highpressure regions, energy input, such as an increase of temperature, must increase entropy but decrease entropy on negative temperature vacuum (Figure 2) [67, 68, 69]. However, the above studies were carried out in crystals and other ordered states and were influenced by gravitational effects.

Entropy is a function of temperature and volume. For the entropy change in ideal gas:

$\Delta S=n R \ln \frac{\boldsymbol{V}}{V_{\text {。 }}}$

Similarly, the entropy change at constant volume:

$\Delta S=n C_{v} \ln \frac{T}{T_{\mathrm{o}}}(8)$

Where $\mathrm{S}$ is entropy, $\mathrm{n}$ is the number of moles, $\mathrm{R}$ is the ideal gas constant.

Although entropy is often an allusive concept, it is often associated with a thermal disorder [70], which might be related to gravitational effects. Recent entropic studies carried out in gravity-free simulations contradict the above expectations; the findings show order increasing entropic effect [4]. Likewise, in computer simulations (where gravity also has no role), entropic effects increase the future degrees of freedom [5]. Notably, both examples enhance the system's ability to do work. 
A possible connection between gravity (the function of temperature), and entropic effects indicates that both increasing and eliminating gravitational effects increase entropy, the latter via order and greater degrees of freedom. Similarly, it would lead to the Tolman temperature gradient. Gravity-free systems' order increasing potential corresponds to work potential. The above conclusion might explain the antigravity behavior of negative temperatures, a possible connection to dark energy [66].

If gravity were to dominate the global structure of the cosmos, we would have globular galactic accumulations with voids converging into corners. In reality, the opposite happens; voids are near-spherical, their expansion pushing galactic structures toward the periphery. Galaxies move towards the densest areas and away from regions with little mass - the voids (relative to the universe's overall expansion) [71, 23]. Like in foam, gravitational structures form walls, knots, and immense size filaments.

The discrete energy compact dimensions formulate entropic effects (Figure 2). These entropic effects might be behind dimensional asymmetry [5, 4, 72].

We can consider the universe as a gas. Compressing volume expands time to infinity, whereas expanding volume rewinds time to zero. Black hole horizons immense field strength slow expansion originating [73]. The vast cosmic voids [22, 74] found by the Sloan Digital Sky Survey $[75,76]$ hide expanding space. For example, the long, fully connected supervoid, Eridanus [77], appears to originate in the CMB cold spot [78].

The cosmos appears to be a harmonic oscillator, alternating between two and four dimensions. The spatial field operates as a clock, tracking the WF evolution $[17,16]$. Time progression from time zero in the white holes to time infinity in the black holes is the function of interaction. In contrast, the volume of space progresses on an opposite trajectory. Space originates in white holes' expansive four-dimensional vacuum, which degrades into a two-dimensional black hole horizon.

Within gravity regions, the field strength increases the speed of interaction. In contrast, expanding areas prohibit interactions (antimatter).

The Hamiltonian of the system:

$H=1 / 2 p^{2} / m+1 / 2 k x^{2}$

where $\mathrm{m}$ is the particle mass, and $\mathrm{k}$ is the stiffness coefficient.

The time-independent Schrödinger equation:

$-1 / 2\left(\hbar^{2} / m\right)\left(d^{2} \psi / d x^{2}\right)+1 / 2 k x^{2} \psi=E \psi(10)$ 
where $\hbar$ is Planck's constant divided by $2 \pi, \mathrm{v}$ is its velocity, and $\psi$ is called the WF for the system. The Fourier transformation solutions

$F(\omega)=\int \exp (-i \omega x) f(x) d x$

Applying the Fourier transform to the time-independent Schrödinger equation for a harmonic oscillator naturally leads to an oscillatory type solution. The peaks in the probability density function can be interpreted as the spectrum of the system.

$\left(\mathrm{d}^{2} \mathrm{~F} / \mathrm{d} \omega^{2}\right)=-\left[2 \mathrm{E} / \mathrm{k}-\left(\hbar^{2} /(\mathrm{km})\right) \omega^{2}\right] \mathrm{F}(\omega)(12)$

The WF of the universe forms either a node or an antinode (voids) at its poles (Figure 2), causing an association between void sizes and clusters of a particular mass scale, for example [39]. Correspondingly, recent calculations have found a power-law scaling relation between the void size and the corresponding cluster mass [79, 80, 81]. The scatter in the scaling relation is more significant at low redshifts and small voids, indicating that larger voids are more insulated from environmental influences (Figure 4).

Current literature vigorously debates the existence and nature of white holes $[41,54,82,83$, 84]. We note that black hole survival is connected to extreme pressures and temperatures. Similarly, negative temperature and vacuum ensure the stability of white holes $[85,77,78$, $79,81]$, within the lowest density, central portions of voids. These regions cause inflation by being protected from gravitational influences [86, 87] (Figure 4, orange regions, Figure 7).

The AdS/CFT correspondence, a holographic mapping, asserts that a lower-dimensional non-gravitational theory can fully describe a higher dimensional gravitational one $[88,38$, 48]. In our derivation, we find that gravitational regions separate high dimensional voids from the low dimensional horizon. When using the CFT side (as opposed to the more usual AdS side) to analyze black holes as a starting point, Almheiri, Marolf, Polchinski, and Sully (AMPS, 2012) found information conservation (i.e., black hole formation and evaporation process are unitary), the firewall hypothesis $[49,66]$.

Although gravity is Lorentz invariant $[89,90]$, the particles' dimensional anisotropy turns gravity into a bipolar force, which leads to the Tolman temperature gradient $[79,80]$. Although three-dimensional space is highly reactant, it is immune to dimensionality transformations. Dimensional disturbances caused by collisions and explosions produce spectacular destructive power [81, 82]. The contrasting influences of the WF and spatial field give rise to global self-regulation that fine-tunes the cosmic parameters and can promote fractal topology with enormous complexity [95].

Considering the symmetries of the WF as an algebraic object defined by its vibration frequencies connects it to the moonshine conjecture. The spatial field curvature can be 
described by a specific trigonometric $\mathrm{j}$-function, which registers the structure of the monster group as time.

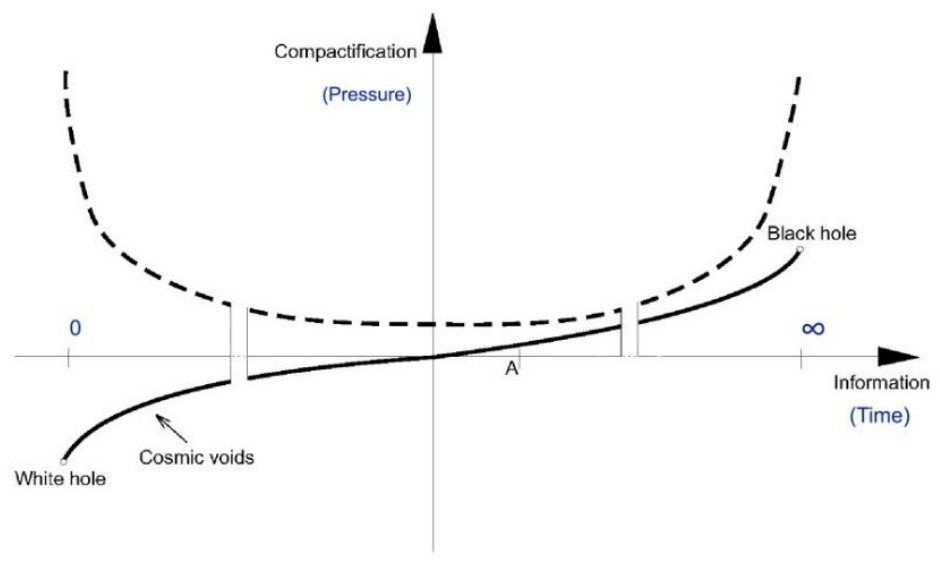

Figure 6. The energy-information changes in the universe The $x$-axis represents the information accumulation or age (from 0 to $\infty$ ). The $y$-axis is the degree of compactification (dimensionality reduction from 4 to 2). The derivative (dotted line) is the Lorentz contraction. A is the gravity on an Earth-like planet.
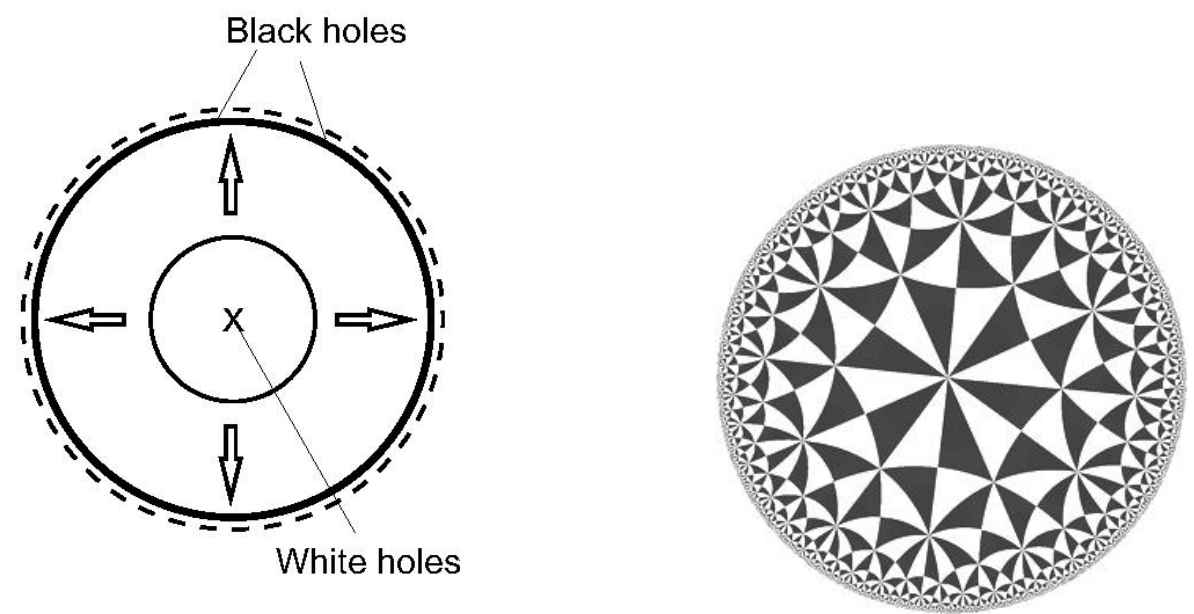

Figure 7. Left: The global topological map of the universe Right: The hyperbolic plane with its inherent self-symmetries satisfying the AdS/CFT conjecture (Wikimedia Commons: Tom Ruen) The immense field strength of the cosmos' outer boundary slows expansion. The vacuum energy of white holes is a negative pressure that expands space into the fourth dimension (hyperbolic geometry). The poles' opposing dynamics (2 and 4 dimensions) enforce constant interactions on the dynamic and unstable three-dimensional regions (marked by white arrows), increasing complexity and engendering evolution. 
Quantum mechanics

As we have seen, antipodal dynamics could very well explain the current structure and curvature of space (i.e., topology) [28, 39]. Interaction between spatial field and WF curves the first and changing the frequencies of the second. The orthogonal transformation between the compact dimensions and the spatial field leads to many well-known consequences of quantum mechanics. Interaction (endothermic or exothermic) preserves inner products in Hilbert space [96], energy conservation (Noether's theorem) implies an inverse relationship between spatial field curvature/volume and ancillary frequencies (Figure 1, Figure 2).

\section{Inertia}

Brans showed that if one adopts the modern geometric interpretation of GR, then the inertial mass of a free test particle cannot change in a gravitational field [2, 3]. In our model, the particle vibrations of string theory are related to the field strength (curvature or metric), satisfying the requirement of Mach's principle.

Because an object's position corresponds to a freely hanging plumb, deviations in the angle of that plumb (location of the object) thereby change the equilibrium of the whole universe and lead to inertia. Intertia is proportional to both the mass of the object and the field strength (i.e., topological distance from the black hole) represented by the metric $g(v, w)$. The effect is congruent with the intuitive expectation that the inertial mass of a test particle increases in the vicinity of a large mass, such as black holes. Therefore, inertia reflects the whole universe's field structure and global dynamics, as Mach had insisted.

An object's position corresponds to a freely hanging plumb (Figure 7). Deviations in the angle of that plumb (location of the object) thereby change the whole universe's equilibrium and lead to inertia, a force proportional to both the mass of the object and the field strength (i.e., topological distance from the black hole). Inertia is most significant in the vicinity of black holes' unapproachable horizons [49].

The horizon problem

In physics, chemistry, and biology, compound or phase change formation often precipitate reactions. The dependence of interaction on the existence of compact dimensions defines time zero as the compact dimension formation. The compact dimensional phase-change allowed space to solidify as the gravity field. The compact dimensions' birth guarantees the identical initial conditions found in the CMB throughout space [56]. The birth of compact dimensions (time zero) guarantees homogeneity throughout space. The uniform and nearly smooth initial distribution found in the $\mathrm{CMB}$ requires a phase change, such as the compact dimensions formation. 


\begin{tabular}{|l|l|}
\hline Compact dimensions & Field of gravity (clock system) \\
\hline Discrete WF & Topological manifold \\
\hline Non-locality, insulated from gravity & locality (forms pressure and curvature) \\
\hline Entanglement is reversible & Interaction is irreversible (stable topology) \\
\hline Constant frequencies increase entropy & Tolman density gradient \\
\hline Evolution via the Schrödinger equation & $\begin{array}{l}\text { The Schrödinger equation describes the global } \\
\text { state }\end{array}$ \\
\hline $\begin{array}{l}\text { Measurement is decoherence of the WF, } \\
\text { changing its frequency }\end{array}$ & $\begin{array}{l}\text { Measurement updates the local volume, } \\
\text { changing the field curvature }\end{array}$ \\
\hline Discrete energy level & Dimensional anisotropy \\
\hline
\end{tabular}

Table I, The orthogonality of space Qualitative differences between compact dimensions and space, permits the cosmos' self-regulation. Global self-regulation, which fine-tunes the cosmos' parameters, requires the orthogonality of space and compact dimensions.

\section{Discussion}

Our proposed hypothesis satisfies the axiomatic formalization of the two leading theories in modern physics, quantum mechanics and GR. Einstein's gravitational theory might be a limiting case of a more general theory in which space is a dynamic variable changing between four dimensions in the white holes and two dimensions in the black holes [35]. The cosmological constant might be a dimensionality function [59]. Field strength and vacuum energy satisfy Newton's third law by forming symmetric forces, which drive cosmic expansion. Recognizing the rich topology of space could lead to a deeper understanding of gravity. The universe's poles explain the accelerating expansion without the need for introducing new forces, such as dark energy and dark matter [41].

The relativistic Dirac equation's universal applicability expresses many qualities of the matter structure. The present model's interpretation of the particle is a black hole-directed movement due to gravitational effects. The possible occurrence of antimatter regions in the baryon-dominated universe has been postulated [97, 98]. However, the antimatter content of voids is nonreactant. 
Newtonian gravity and GR are successful in describing our three-dimensional experience within the solar system. Our hypothesis connects inertial mass to the global topology [99]. Therefore $\mathrm{PaW}$, which originates in the microstructure of space, leads to dimensional anisotropy. The hypothesis can reproduce the relativity of motion and an accelerating universe without exotic particles or forces.

In the Machian view, the global mass distribution must determine the inertial frame of reference, the inertial mass, and acceleration [91]. Our model satisfies Mach's principle and eliminates much uncertainty around the Standard Model. Our visual, intuitive, and straightforward framework shows a close fit with large-scale observations. It can provide new considerations for understanding the cosmological constant and the coincidence problem (matter density depends on dimensionality). The three-dimensional Euclidean space represents a dynamic birthplace for the formation of stars, planets, and life forms.

Recently, Lucia and Grisolia [44] uncovered time as a function of frequency, $t=1 / v$. Their extensive calculations of time intervals' values concerning temperature support our argument on gravity's connection to entanglement. The irreversible thermodynamics might lead to the irreversibility of time [42] and the existence of negative absolute temperatures [65]. In this way, time is defined by entropy generation and the entropy rate.

The field curvature generates the universe's large-scale structure by producing antipodal sinks and sources. These sinks and sources can explain three unexplained puzzles in physics: the so far unexplained behavior of (1) cold vacuum in the lab, (2) the expansion of space eliminates gravity, creating order increasing entropic effects, the potential for work (via order and degree of freedom), (3) the accelerating expansion originating in cold cosmic voids, and (4) a tight power-law scaling relation between the void size and the corresponding cluster mass [79].

\section{Summary}

We constructed a model universe by equipping a field with compact dimensions. We find that our very simple clock system had far-reaching consequences for interaction, entropy, and the global state of the cosmos. The insulated WF (string theory) is necessary and sufficient to generate stable spatial curvature (GR). GR represents a clock system, and quantum mechanics and string theory describe the WF. Interaction between the field and the WF triggers a global evolution, where the curvature represents the particle's WF's evolution as time. This way, the global shape of the universe, which satisfies fundamental conservation principles, occurs according to the Page and Wootter mechanism. The resulting system satisfies Mach's principle and replicates the qualities of our physical world, such as quantum mechanics, relativity, and accelerating expansion. The interdependence of compact 
dimensions and spatial topology leads to self-regulation, which can fine-tune the universe's parameters.

The entropic disorder is apparently related to gravitational effects. Both increasing and eliminating gravitational effects increase entropy. Eliminating gravity permits order and degrees of freedom increasing transformations. The relationship may explain negative temperatures' antigravity-like effects. The ability of gravity-free systems to spontaneously increase work potential may give Maxwell's demon the last laugh.

Physics has a solid foundation, which should not be changed at a whim. Nevertheless, the persistent questions at the core of relativity (dark matter and dark energy), cosmology (the horizon problem and inflation), and quantum mechanics urges us to reconsider fundamental questions about the above areas. Our work might serve just a first baby step.

The thermodynamics of interaction gives rise to a global temporal evolution, creating poles with dimensionality differences (four-dimensional white holes and two-dimensional black hole horizons). Sudden dimensionality modifications might cause the destructive power of explosions and collisions. Space's topology and its accelerating expansion may originate in the WF's evolution rather than dark energy and dark matter. The anisotropic universe satisfies both PaW and Mach's principles, and it causes the Tolman temperature gradient. According to GR, massive objects initiate converging light paths, but light rays experience divergence when passing through cosmic voids. The above considerations reproduce the qualities of our physical world (quantum mechanics, relativity, and accelerating expansion). Although the details of our hypothesis still need to be worked out, computer simulations and negative temperature experiments in a microgravity environment can verify its points.

\section{Declarations}

The work received no funding. The authors declare no conflict of interest. 


\section{Works Cited}

[1] R. Laymon, "Newton's Bucket Experiment," Journal of the History of Philosophy, pp. 16, 399 - 413., 1978.

[2] C. Brans, " Mach's Principle and a Relativistic Theory of Gravitation," II Phys. Rev. , pp. $125,388,1962$.

[3] C. Brans, "Absence of Inertial Induction in General Relativity," Phys. Rev. Lett., Vols. 39, 856, pp. 39, 856, 1977.

[4] A. Haji-Akbari, M. Engel, A. Keys, X. Zheng, R. Petschek, P. Palffy-Muhoray and S. Glotzer, "Disordered, quasicrystalline and crystalline phases of densely packed tetrahedra," Vols. 462, 773-777, 2009.

[5] A. D. Wissner-Gross and C. E. Freer, "Causal Entropic Forces.," Phys. Rev. Lett., no. doi: 10.1103/PhysRevLett.110.168702, p. 110:168702, 2013.

[6] D. N. Page and W. K. Wootters, PhysRevD., pp. 2885, 10.1103/27.2885., 1983.

[7] E. Deli, The Science of Consciousness; How a new understanding of space and time infers the evolution of the mind, US, 2015.

[8] B. S. DeWitt, "," Phys. Rev. , pp. 160, 1113. 10.1103/PhysRev.160.1113., 1967.

[9] E. Moreva, G. Brida, M. Gramegna and e. al., Phys. Rev. A, vol. 89, no. 052122, 2014.

[10] W. Wootters, " "Time" replaced by quantum correlations," Int. J. Theor. Phys., pp. 23, 701-711, 1984.

[11] C. Foti, A. Coppo and G. e. a. Barni, "Time and classical equations of motion from quantum entanglement via the Page and Wootters mechanism with generalized coherent states.," no. 12, 1787, 2021.

[12] M. Woods, "The Page-Wootters mechanism 36 years on: a consistent formulation which accounts for interacting systems.," Vols. Quantum Views 3, 16, 2019.

[13] R. Gambini and J. Pullin, "The Montevideo Interpretation of Quantum Mechanics: A Short Review.," Entropy, p. 20, 2018. 
[14] M. Rotondo and Y. Nambu, " Clock Time in Quantum Cosmology," arXiv: General Relativity and Quantum Cosmology, 2019.

[15] F. O. G. \&. R.-G. D. Lobo, "Microscopic wormholes and the geometry of entanglement.," Eur. Phys. J. C, vol. 74, no. 2924, 2014.

[16] A. R. H. a. A. M. Smith, "Quantum," Vols. 3, 160, no. 10.22331/q-2019-07-08-160. , 2019.

[17] A. Smith and M. Ahmadi, "Quantizing time: Interacting clocks and systems," arXiv: Quantum Physics., vol. arXiv: Quantum Physics., 2017.

[18] I. Etxebarria, M. Montero, K. Sousa and Valenzuela, "Nothing is certain in string compactifications," arXiv: 2005.06494., 2020.

[19] L. A. Maccone, "Fundamental Problem in Quantizing General Relativity," Found. Phys. , vol. 49, p. 1-10, 2019.

[20] R. Weygaert, "Voids and the Cosmic Web: cosmic depression \& spatial complexity," arXiv: Cosmology and Nongalactic Astrophysics, pp. 11, 493-523, 2014.

[21] A. Kravtsov, "On the Origin of the Global Schmidt Law of Star Formation," The Astrophysical Journal, p. 590, 2003.

[22] R. B. Tully, H. Courtois, Y. Hoffman and D. Pomarède, "The Laniakea supercluster of galaxies," Vols. 513 (7516), 71-3. , 2014.

[23] R. Tully, D. Pomarède, R. Graziani, H. Courtois, Y. Hoffman and E. Shaya, "Cosmicflows-3: Cosmography of the Local Void.," arXiv: Cosmology and Nongalactic Astrophysics., 2019.

[24] U. Lindner, J. Einasto, M. Einasto, W. Freudling, K. Fricke and E. Tago, " "The structure of supervoids. I. Void hierarchy in the Northern Local Supervoid"," Astron. Astrophys. , p. 301: 329, 1995.

[25] R. Freedman and W. Kaufmann III, Stars and galaxies: Universe., New York City: W.H. Freeman and Company., 2008.

[26] S. Gregory and L. Thompson, "The COMA / A 1367 Supercluster and Its Environs.," Astrophysical Journal, pp. 222, 784-799, 1978. 
[27] J.-R. Pycke and E. Russell, "A new statistical perspective to the cosmic void distribution," ApJ , p. 821 110, 2016.

[28] J. a. G. W. W. Wheeler, Black Holes, and Quantum Foam p. 235, Norton \& Company, 2000.

[29] K. Borsuk, "Drei sätze uber di n-dimensionale euklidische sphäre," Fund. Math. , vol. XXX, pp. 177-196, 1933.

[30] J. F. Peters, Computational Proximity, Switzerland: Springer, 2016.

[31] A. Di Concilio, C. Guadagni, J. Peters and S. Ramanna, "Descriptive proximities. Properties and interplay between classical proximities and overlap.," Math. in Comp. Sci., vol. 12, no. 1, pp. 91-106, 2018.

[32] J. M. J. B. A. a. Z. G. Matoušek, in Using the Borsuk-Ulam Theorem: Lectures on Topological Methods in Combinatorics and Geometry, Springer, 2003.

[33] J. F. Peters, "Ribbon complexes and their aproximate descriptive proximities. Ribbon and vortex nerves, Betti numbers and planar divisions.," Bulletin of Allahabad Math Society, vol. 35, no. 1, pp. 1-14, 2020.

[34] A. e. b. J. P. Tozzi, "Borsuk-Ulam Theorem Extended to Hyperbolic Spaces," in Proximity. Excursions in the Topology of Digital Images, doi:10.1007/978-3-31930262-1., 2016, p. 169-171..

[35] J. R. (. E. M. D. Weeks, "Chapter 14: The Hypersphere," in The Shape of Space: how to visualize surfaces and three-dimensional manifolds. , ISBN 978-0-8247-7437-0, 1985.

[36] G. Marsaglia, "Choosing a point from the surface of a sphere.," Ann Math Stat., vol. 43, p. 645-6., 1972.

[37] D. Anosov, "Diffeomorphism," in Encyclopedia of Mathematics, Kluwer Academic Publishers, 1995, pp. 155-156..

[38] L. a. F. A. Susskind, Quantum Mechanics. The Theoretical Minimum, UK: Penguin Random House ISBN 97-0-141-97781-2., 2014.

[39] L. Martyushev and E. Shaiapin, "From an Entropic Measure of Time to Laws of Motion.," Entropy, p. 21, 2019. 
[40] L. Martyushev, "On Interrelation of Time and Entropy," Entropy, pp. 19, 345, 2017.

[41] R. Kastner and S. Kauffman, "Are Dark Energy and Dark Matter Different Aspects of the Same Physical Process?," vol. https://doi.org/10.3389/fphy.2018.00071 , 2018.

[42] A. Chatterjee and G. Iannacchione, "Time and Thermodynamics Extended Discussion on "Time \& clocks: A thermodynamic approach"," 2020.

[43] E. Verlider, "On the origin of gravity and the laws of Newton," J. High Energ. Phys., pp. 4, 29, 2011.

[44] U. Lucia and G. Grisolia, "Time \& clocks: A thermodynamic approach," Vols. 16, 102977, 2020.

[45] U. Lucia, G. Grisolia and A. Kuzemsky, "Time, Irreversibility and Entropy Production in Nonequilibrium Systems," vol. 22, 2020.

[46] R. Landauer, "Irreversibility and heat generation in the computing process," IBM Journal of Research and Development, vol. 5 , no. 3, p. 183-191, 1961.

[47] Y. C. M. a. C. C. J. Bardoux, "Conformally coupled scalar black holes admit a flat horizon due to axionic charge.," High Energ. Phys., vol. 8, 2012.

[48] J. M. Maldacena, "The Large N Limit of Superconformal Field Theories and Supergravity," vol. 2: 231-252, 1998.

[49] A. Almheiri, D. Marolf, J. Polchinski and J. Sully, "Black holes: Complementary or Firewalls?," ArXiv: 1207. 3123v4 \{hep-th\}, 2012.

[50] Saulnier et al., "A study of generation and rupture of soap films," vol. 10:2899-2906. , 2014.

[51] B. Ahmadi, S. Salimi and A. Khorashad, "Irreversible Work, Maxwell's Demon and Quantum Thermodynamic Force.," 2018.

[52] Johnson, "Thermodynamic volumes for AdS-Taub-NUT and AdS-Taub-Bolt.," Vols. 31, 235003, 2014.

[53] P. Wesson, "Fundamental Unsolved Problems in Astrophysics.," Space Science Reviews, vol. 98, no. 329342, 2001. 
[54] J. V. A. R. K. a. D. N. Narlikar, "High energy radiation from white holes," Vols. 251, 591, 1974.

[55] S. E. Rugh and H. Zinkernagel, Vols. 33, 663, 2002.

[56] R. Weygaert, "Voronoi Tessellations and the Cosmic Web: Spatial Patterns and Clustering across the Universe The cosmic web: geometric analysis," Astrophys., vol. 1, p. $070708.1441 ., 2007$.

[57] S. Cantalupo, F. Arrigoni-Battaia and J. e. a. Prochaska, "A cosmic web filament revealed in Lyman- $\alpha$ emission around a luminous high-redshift quasar.," Nature, vol. 506, no. 7486, p. 63-66, 2014.

[58] J. S'anchez-Monroy and C. Quimbay, "Cosmological Constant in a Quantum Fluid Model," Vols. 20, 2497-2506, 2011.

[59] D. Lovelock, "The Einstein Tensor and Its Generalizations," Journal of Mathematical Physics, vol. 12, pp. 3, 498-501,, 1971.

[60] S. Braun, J. P. Ronzheimer and M. e. a. Schreiber, "Negative Absolute Temperature for Motional Degrees of Freedom," Science, vol. 339, no. 6115, p. 52-55., 2013.

[61] S. Calabrese and A. Porporato, "Origin of Negative Temperatures in Systems Interacting with External Fields.," 2018.

[62] B. Lavenda, "Do 'negative' temperatures exist?," vol. 32(23):4279, 1999.

[63] F. Miceli, M. Baldovin and A. Vulpiani, "Statistical mechanics of systems with longrange interactions and negative absolute temperature," Vols. 99 4-1, 042152 , 2019.

[64] Z. Merali, "Quantum gas goes below absolute zero," 2013.

[65] N. F. Ramsey, "Thermodynamics and statistical mechanics at negative absolute temperatures.," vol. 103(1):20, 1956.

[66] E. Abraham and O. Penrose, "Physics of negative absolute temperatures," Vols. 95 $1-1,012125,2017$.

[67] M. Baldovin, A. Puglisi, A. Sarracino and A. Vulpiani, "About thermometers and temperature," vol. 113202., 2017. 
[68] M. Baldovin, "Negative Temperature Out of Equilibrium.," Springer, Cham. https://doi.org/10.1007/978-3-030-51170-8_5, In: Statistical Mechanics of Hamiltonian Systems with Bounded Kinetic Terms. Springer Theses, 2020.

[69] L. Onsager, ""Statistical Hydrodynamics"," Il Nuovo Cimento. Nuovo Cim., p. 6(Suppl 2) (2): 279-287., 1949.

[70] M. Kostic, "The Elusive Nature of Entropy and Its Physical Meaning," Entropy, pp. 16, 953-967, 2014.

[71] R. B. Tully, H. M. Courtois and J. G. Sorce, "COSMICFLOWS-3," AJ, pp. 152, 50, 2016.

[72] M. Gogberashvili and I. Kanatchikov, "Machian origin of the entropic gravity and cosmic acceleration.," Int. J. Theor. Phys., pp. DOI: 10.1007/s10773-011- 0971-z., 2011.

[73] J. a. T. A. Peters, "Quantum entanglement on a hypersphere," Int. J. of Theoretical Physics, vol. 55, no. 8, pp. 3689-3696, 2016.

[74] D. G. Lambas, M. Lares and Ceccarelli, "The sparkling Universe: the coherent motions of cosmic voids," Vols. 455, L99, 2016.

[75] D. S. Aguado and e. al., "Sloan Digital Sky Survey," arXiv:1812.02759 [astroph.IM], no. (SDSS-IV Overview), 2018.

[76] R. Adam and e. al., "Planck 2015 results. I. Overview of products and scientific results.," Astron. Astrophys., no. A1., p. 594, 2016.

[77] A. Kovács and J. García-Bellido, "Cosmic troublemakers: the Cold Spot, the Eridanus supervoid, and the Great Walls.," Monthly Notices of the Royal Astronomical Society, pp. 462, 1882-1893., 2016.

[78] M. Farhang and M. Movahed, "CMB Cold Spot in the Planck light.," arXiv: Cosmology and Nongalactic Astrophysics., 2020.

[79] J. Santiago and M. Visser, "Tolman temperature gradients in a gravitational field," arXiv:1803.04106 [gr-qc], 2018.

[80] R. C. Tolman, "On the weight of heat and thermal equilibrium in GR," Phys. Rev., vol. 35 , no. 904, 1930. 
[81] D. W. G. S. K. \&. R. S. A. NOID, "Fractal behavior in classical collisional energy transfer," J. Chem. Phys. , vol. 51, pp. 363-383., 1986.

[82] Y. B. G. L. V. a. M. G. Zeldovich, The Mathematical Theory of Combustion and Explosions., New York: Plenum, 1985.

[83] J. a. T. A. Peters, "String-Based Borsuk-Ulam Theorem," arXiv:1606.04031 , 2016.

[84] L. Rio, J. Åberg, R. Renner, O. Dahlsten and V. Vedral, "The thermodynamic meaning of negative entropy," Vols. 476, 476-476, 2011.

[85] P. S. a. N. R. Joshi, "Black hole paradoxes," Journal of Physics, no. Conference Series $759,2014$.

[86] D. Lovelock, "The Einstein Tensor and Its Generalizations," Journal of Mathematical Physics, vol. 12, no. 3, p. 498-501, 1971.

[87] J. Shim, C. Park, J. Kim and H. Hwang, "Identification of Cosmic Voids as Massive Cluster Counterparts.," ApJ , p. 908. 211, 2021.

[88] A. Pontzen, A. Slosar, N. Roth and H. Peiris, " Inverted initial conditions: Exploring the growth of cosmic structure and voids," Physical Review D, pp. 93, 103519, 2016.

[89] W. A. Hellwing, M. Cautun, R. van de Weygaert and B. T. Jones, "Caught in the cosmic web: Environmental effect on halo concentrations, shape, and spin.," Phys. Rev. D , pp. 103, 063517, 2021.

[90] A. Lightman and D. Eardley, "Black Holes in Binary Systems: Instability of Disk Accretion.," The Astrophysical Journal, p. 187, 1974.

[91] R. Wald and S. Ramaswamy, "Particle production by white holes," Physical Review $D$, pp. 21, 2736-2741, 1980.

[92] E. Bianchi, M. Christodoulou, F. D'Ambrosio, H. Haggard and C. Rovelli, "White Holes as Remnants: A Surprising Scenario for the End of a Black Hole.," 2018.

[93] D. Frenkel and P. B. Warren, "Gibbs, Boltzmann, and negative temperatures.," Am. J. Phys., vol. 83, no. 163, 2015.

[94] S. Lee, "A solution to the initial condition problems of inflation : NATON.," 2019.

[95] J. B. C. \&. L. A. Vieira, "Cosmology with Negative Absolute Temperatures," 2016. 
[96] G. 't Hooft, "Dimensional reduction in quantum gravity," 1993.

[97] M. Hohensee and e. al., "Limits on violations of Lorentz symmetry and the Einstein equivalence principle using radio-frequency spectroscopy of atomic dysprosium.," Physical Review Letters., vol. 5, no. 050401., p. 111, 2013.

[98] S. Peck and e. al., "New Limits on Local Lorentz Invariance in Mercury and Cesium.," Physical Review A., vol. 1, no. 012109. , p. 86, 2012.

[99] S. Wolfram, A new kind of Science, Wolfram Research , 2002.

[100] C. S. Frenk and S. D. M. White, "Dark matter and cosmic structure," arxiv.org/abs/1210.0544, 1-27., 2012.

[101] M. Khlopov, S. Rubin and A. Sakharov, "Possible Origin of Antimatter Regions in the Baryon Dominated Universe," Physical Review D, pp. 62, 083505, 2000.

[102] M. Khlopov and A. Kirichenko, "Anihelium flux from antimatter globular cluster," arXiv: High Energy Astrophysical Phenomena, 2020.

[103] N. F. Ramsey, "Thermodynamics and statistical mechanics at negative absolute temperatures," Physical Review , p. 103.1. 20., 1956. 